\title{
STRONGLY NONLINEAR ELLIPTIC VARIATIONAL INEQUALITIES IN UNBOUNDED DOMAINS
}

\author{
VESA MUSTONEN
}

\section{Introduction}

In this paper we are concerned with the solvability of strongly nonlinear variational inequalities involving differential expressions of the form

$$
A u(x)+B u(x), x \in \Omega,
$$

where $\Omega$ is a (unbounded) domain in $\mathbb{R}^{N}(N \geqq 2)$,

$$
A u(x)=\sum_{|\alpha| \leqq k}(-1)^{|\alpha|} D^{\alpha} A_{\alpha}\left(x, u(x), \ldots, D^{k} u(x)\right)
$$

is quasilinear,

$$
B u(x)=\sum_{|\beta| \leqq m}(-1)^{|\beta|} D^{\beta} B_{\beta}\left(x, u(x), \ldots, D^{m} u(x)\right)
$$

is strongly nonlinear in the sense that $B_{\beta}$ do not satisfy a polynomial growth condition, and $0 \leqq m<k<N$. Two particular methods in tackling these problems are available: 1) the use of mappings of monotone type, which may not be everywhere defined on the Sobolev spaces involved; 2) the theory of monotone operators acting on an Orlicz space; (see [6] and numerous references listed therein). We shall consider here the solvability by means of either methods.

Our study by the method 1) rests on the foundation of the recent papers of Hess [12], Các [4] and Edmunds, Moscatelli and Webb [9]. Hess introduced a new class of maps of monotone type for which the solvability can be shown under a mild coercivity condition. However, this result seems to be directly applicable for a rather restricted class of expressions $A$ only (for example for a linear one). The treatment of Các reaches a more extensive class of expressions $A$ but stronger conditions for $B$ and for coercivity are needed. Moreover, the proof involves certain concrete function spaces and differential expressions. We prove here the corresponding result in an abstract form by adopting the concepts introduced by Hess with a mild coercivity condition. We apply the solvability theorems obtained to variational inequalities generated by (1) in unbounded domains. We extend and 
generalise the corresponding results of [9]. In particular, the $\varepsilon$-condition (see $\left(\mathbf{B}_{4}\right)$ in $\S 4$ ) is removed when $A$ is linear, and the requirement that the origin is an interior point in the convex set involved is relaxed also when $A$ is a quasilinear operator. Moreover, our coercivity conditions are weaker than those occurring in [9]. In [4] and [12] the applications are given in bounded domains only.

On the other hand, we show here that in certain particular cases the results obtained by the method 1) can be improved by means of the method 2). Our Orlicz space method for the spaces involving unbounded domains is established by the recent embedding theorems obtained by Edmunds and Evans [7], [8]. In particular, if the treatment given here is applied to a strongly nonlinear elliptic Dirichlet problem

where

$$
\left\{\begin{array}{l}
A u(x)+B u(x)=F(x) \text { in } \Omega, \\
D^{\alpha} u(x)=0 \text { on } \partial \Omega \text { for all }|\alpha| \leqq k-1,
\end{array}\right.
$$

$$
A u(x)=\sum_{|\alpha|,|\beta| \leqq k}(-1)^{\alpha} D^{\alpha}\left(a_{\alpha \beta}(x) D^{\beta} u(x)\right)
$$

is linear, $B$ is given by (3), $F$ is a prescribed function in $L^{2}(\Omega)$ and $0 \leqq m<k<N$ with $k-m=N / 2$, then the results obtained in [6], [8] for the existence of a weak solution, can be generalised and improved.

\section{Prerequisites}

Let $\Omega$ be an open subset of $\mathbf{R}^{N}(N \geqq 2)$ with Lebesgue measure $d x$, and let $C_{0}^{k}(\Omega)$ stand for the space of all real-valued functions which are $k$ times continuously differentiable on $\Omega$ and have compact support in $\Omega$. By $H_{0}^{k, p}(\Omega)$ we shall mean the completion of $C_{0}^{k}(\Omega)$ with respect to the norm

$$
\|u\|_{k, p}=\sum_{i=0}^{k}\left\|D^{i} u\right\|_{p}
$$

with $\left|D^{i} u(x)\right|^{2}=\sum_{|\alpha|=i}\left|D^{\alpha} u(x)\right|^{2}$, where the summation extends over all $N$-tuples $\alpha=\left(\alpha_{1}, \ldots, \alpha_{N}\right)$ of non-negative integers with $|\alpha|=\alpha_{1}+\ldots+\alpha_{N}$ and $D^{\alpha}=$ $=\prod_{j=1}^{N}\left(\partial / \partial x_{j}\right)^{\alpha_{j}}$. For $1<p<\infty, H_{0}^{k, p}(\Omega)$ becomes a separable, reflexive Banach space. In particular, $H_{0}^{k, 2}(\Omega)$ is a Hilbert space with a familiar inner product.

Let $B(x, R)$ denote the open ball in $\mathbf{R}^{N}$ with centre $x$ and radius $R$, and given any $\mu>0$ and any measurable function $Q$ denote

where

$$
M_{\mu}(|Q|)=\sup _{x \in \Omega} \int_{\Omega \cap B(x, 1)}|Q(y)| w_{\mu}(x-y) d y,
$$

$$
w_{\mu}(x)=\left\{\begin{array}{l}
|x|^{\mu-N} \text { if } \mu<N \\
1 \text { if } \mu>N \text { or } \mu=N \text { and }|x|>1 \\
1-\log |x| \text { if } \mu=N \text { and }|x| \leqq 1 .
\end{array}\right.
$$


We also denote

$$
Q_{R}(x)=\left\{\begin{array}{c}
Q(x) \text { if }|x|>R \\
0 \quad \text { otherwise }
\end{array}\right.
$$

An Orlicz function is any map $\Phi: \mathbf{R} \rightarrow \mathbf{R}$ which is convex, continuous, even and for which

$$
\lim _{r \rightarrow 0} \frac{\Phi(r)}{r}=0 ; \quad \lim _{r \rightarrow \infty} \frac{\Phi(r)}{r}=\infty .
$$

The Orlicz class $K_{\Phi}(\Omega)$ is defined as the set of (equivalence classes of) real-valued measurable functions $u$ such that

$$
\int_{\Omega} \Phi[u(x)] d x<\infty,
$$

and the Orlicz space $L_{\Phi}(\Omega)$ as a linear hull of $K_{\Phi}(\Omega)$ furnished with the Luxemburg norm

$$
\|u\|_{\Phi}=\inf \left\{\lambda: \int_{\Omega} \Phi[u(x) / \lambda] d x \leqq 1\right\} .
$$

The closure in $L_{\Phi}(\Omega)$ of the bounded functions with compact support in $\bar{\Omega}$ is denoted by $E_{\Phi}(\Omega)$. An Orlicz function $\Phi$ is said to satisfy the $\Delta^{2}$-condition if there exist $\lambda>1$ and $r_{0}>0$ such that $\Phi^{2}(r) \leqq \Phi(\lambda r)$ for all $r \geqq r_{0}$. For example, $\Phi(r)=|r|^{\varrho} e^{|r|^{\delta}}$ with $\varrho>1$ and $\delta>0$ satisfies the $\Delta^{2}$-condition. If $\Phi$ and $\Psi$ are two Orlicz functions, we shall write $\Psi \prec \Phi$ at 0 or $\infty$, if for all $\lambda>0$,

$$
\lim _{r \rightarrow 0} \frac{\Phi(\lambda r)}{\Psi(r)}=\infty, \quad \text { or } \quad \lim _{r \rightarrow \infty} \frac{\Phi(\lambda r)}{\Psi(r)}=\infty,
$$

respectively. If $\Psi \prec \Phi$ at 0 and $\infty$, then for any $\varepsilon>0$ there is a constant $K(\varepsilon)>0$ such that $\Psi(r) \leqq K(\varepsilon) \Phi(\varepsilon r)$ for all $r \geqq 0$. For a more detailed treatment of Orlicz spaces we refer to [14].

Finally, the following notations will also be used. The number of $\alpha$ 's with $|\alpha| \leqq m$ is denoted by $s_{m}$. By $\xi_{m}$ we denote a typical vector in $\mathbf{R}^{s_{m}}$. The components of $\xi_{m}$ are denoted by $\xi_{\alpha}$ so that $\xi_{m}=\left(\xi_{\alpha}\right)_{|\alpha| \leqq m}$. Furthermore, $\xi_{m}(u)(x)=\left\{D^{\alpha} u(x):|\alpha| \leqq m\right\}$. If $V$ is a real normed space, its dual space is denoted by $V^{*}$, and $\langle.,$.$\rangle stands for$ the natural pairing between $V$ and $V^{*}$.

\section{Method involving operators of monotone type}

Let $V$ be a real reflexive Banach space and let $W$ be a normed space with $W \subset V$; the natural injection $i$ is supposed to be continuous. Let $V_{1}$ be a subset of $V$ such that $W \subset V_{1} \subset V$. We set the following conditions for the map $T: V_{1} \rightarrow W^{*}$ (cf. [12]).

$\left(\mathrm{A}_{1}\right) \quad T$ is continuous from finite dimensional subspaces of $W$ to the weak ${ }^{*}$-topology of $W^{*}$; 
$\left(\mathrm{A}_{2}\right)$ if $\left(u_{n}\right)$ is any sequence in $W$ with $u_{n} \rightarrow u$ weakly in $V$ and $\left\langle T u_{n}, u_{n}\right\rangle \leqq$ const. for all $n$, then $u \in V_{1}$ and $T u_{n} \rightarrow T u$ in weak*-topology of $W^{*}$;

$\left(\mathrm{A}_{3}\right)$ to $u \in V_{1}$ there is associated a real number $\lambda(u)$ such that $\lambda(u) \leqq$ $\leqq \lim \inf \left\langle T u_{n}, u_{n}\right\rangle$ for any sequence $\left(u_{n}\right)$ occurring in $\left(\mathrm{A}_{2}\right)$;

$\left(\mathrm{A}_{4}\right) \lim \sup \left\langle T u, u_{n}\right\rangle \leqq \lambda(u)$ for any sequence $\left(u_{n}\right)$ occurring in $\left(\mathrm{A}_{2}\right)$. Hess [12] proves the following

Theorem 3.1. Let $T: V \rightarrow W^{*}$ satisfy the conditions $\left(\mathrm{A}_{1}\right)-\left(\mathrm{A}_{3}\right)$ and let $K$ be a closed convex subset of $V$ containing the origin. If for a given $f \in V^{*}$ and for some $R>0$,

$$
\langle T w-f, w\rangle>0 \text { for all } w \in K \cap W, \quad\|w\|_{V}=R,
$$

then the variational inequality

$$
\langle T u, w\rangle-\lambda(u) \geqq\langle f, w-u\rangle \text { for all } w \in K \cap W,
$$

admits a solution $u$ in $K \cap V_{1}$.

Let $S$ be a map from $V$ to $V^{*}$ and let $\tilde{S}: V_{1} \rightarrow W^{*}$ be defined by $\langle\tilde{S} u, w\rangle=$ $=\left\langle i^{*} S u, w\right\rangle=\langle S u, i w\rangle, u \in V_{1}, w \in W$. The maps we shall be dealing with are of the form $T=\tilde{S}+T_{1}$, where $T_{1}$ satisfies $\left(\mathrm{A}_{1}\right)-\left(\mathrm{A}_{3}\right)$ or $\left(\mathrm{A}_{1}\right)-\left(\mathrm{A}_{4}\right)$, respectively. If the conditions $\left(\mathrm{A}_{1}\right)-\left(\mathrm{A}_{3}\right)$ hold for $T_{1}$ with $u \rightarrow \lambda_{1}(u)$ occurring in $\left(\mathrm{A}_{3}\right)$, then for any bounded, monotone and weakly continuous map $S$ also $T=\widetilde{S}+T_{1}$ satisfies the conditions $\left(\mathrm{A}_{1}\right)-\left(\mathrm{A}_{3}\right)$ with $\lambda(u)=\lambda_{1}(u)+\langle S u, u\rangle$. In particular we have (cf. [12])

Corollary 3.2. Let $T_{1}: V_{1} \rightarrow W^{*}$ satisfy the conditions $\left(\mathrm{A}_{1}\right)-\left(\mathrm{A}_{3}\right)$, let $S: V \rightarrow V^{*}$ be linear, bounded and monotone, and let $K$ be a closed convex subset of $V$ containing the origin. If (3.1) holds for a given $f \in V^{*}$ and for some $R>0$, then the variational inequality

$$
\left\langle T_{1} u, w\right\rangle+\langle S u, w-u\rangle-\lambda_{1}(u) \geqq\langle f, w-u\rangle \text { for all } w \in K \cap W,
$$

admits a solution $u$ in $K \cap V_{1}$.

The proof of the following theorem is essentially a combination of the proofs of Theorem 1 of [12] and Theorem 3 of [4]. We recall first that a map $S: V \rightarrow V^{*}$ is said to be pseudomonotone, if it is bounded and if for any sequence $\left(v_{n}\right)$ in $V$ which converges weakly to $v$ with $\lim \sup \left\langle S v_{n}, v_{n}-v\right\rangle \leqq 0, \lim \inf \left\langle S v_{n}, v_{n}-u\right\rangle \geqq\langle S v, v-u\rangle$ for all $u \in V$.

Theorem 3.3. Let $T_{1}: V_{1} \rightarrow W^{*}$ satisfy the conditions $\left(\mathrm{A}_{1}\right)-\left(\mathrm{A}_{4}\right)$, let $S: V \rightarrow V^{*}$ be pseudomonotone, and let $K$ be a closed convex subset of $V$ containing the origin. If (3.1) holds for a given $f \in V^{*}$ and for some $R>0$, then the variational inequality

$$
\left\langle T_{1} u, w\right\rangle+\langle S u, w-u\rangle-\lambda_{1}(u) \geqq\langle f, w-u\rangle \text { for all } w \in K \cap W,
$$

admits a solution $u$ in $K \cap V_{1}$. 
Proof. Pseudomonotone maps are known to be demicontinuous (see [15], p. 179) so that $T=\tilde{S}+T_{1}$ satisfies $\left(\mathrm{A}_{1}\right)$. Let $\mathscr{F}$ be the set of all finite dimensional subspaces $F$ of $W$ equipped with the norms $\|\cdot\|_{F}=\|\cdot\|_{V}$. Let $j_{F}$ stand for the natural injection of $F$ to $W$ and $j_{F}^{*}$ for its adjoint: $W^{*} \rightarrow F^{*}$. Then $T_{F}=j_{F}^{*} \circ T \circ j_{F}$ is a continuous map from $F$ to $F^{*}$. By (3.1),

$$
\left\langle T_{F} w-j_{F}^{*} f, w\right\rangle>0 \text { for all } w \in F \cap K, \quad\|w\|_{F}=R,
$$

for a given $f \in V^{*}$ and for some $R>0$. By a result for finite dimensional spaces (see [1], [12]) there is $u_{F} \in F \cap K$ with $\left\|u_{F}\right\| \leqq R$ such that

$$
\left\langle T u_{F}, w-u_{F}\right\rangle \geqq\left\langle f, w-u_{F}\right\rangle \text { for all } w \in F \cap K .
$$

For any $F^{\prime} \in \mathscr{F}$ set

$$
U_{F^{\prime}}=\left\{u_{F}: F \in \mathscr{F}, F^{\prime} \subset F, u_{F} \text { as in (3.4) }\right\} .
$$

The family $\left\{U_{F^{\prime}}\right\}_{F^{\prime} \in \mathscr{F}}$ obviously has the finite intersection property. By reflexivity of $V, \cap_{F^{\prime} \in \mathscr{F}}\left\{\right.$ weak cl $\left._{V}\left(U_{F^{\prime}}\right)\right\} \neq \emptyset$. Let $u$ be an element in this intersection. We shall show that $u$ solves (3.3). It is readily seen that $u \in K,\|u\|_{V} \leqq R$, and that the correspondence $F \rightarrow\left\{u_{F}\right\}$ given by (3.4) meets the conditions of Proposition 11 of [3].

Let $w_{0} \in K \cap W$ be arbitrary and let $F_{0}$ be a finite dimensional subspace of $W$ containing $w_{0}$. Then there exists an increasing sequence $\left\{F_{k}\right\} \subset \mathscr{F}$ with $F_{0} \subset F_{1} \subset \ldots$, and for each $k$ an element $u_{k}=u_{F_{k}}$ in $U_{F_{0}}, u_{k} \in F_{k} \cap K$ such that $u_{k} \rightarrow u$ weakly in $V$. Taking account of (3.4) we obtain

$$
\left\langle T u_{n}, w-u_{n}\right\rangle \geqq\left\langle f, w-u_{n}\right\rangle \text { for all } w \in F_{n} \cap K .
$$

In particular,

$$
\left\langle T u_{n}, u_{m}-u_{n}\right\rangle \geqq\left\langle f, u_{m}-u_{n}\right\rangle \text { for } 1 \leqq m \leqq n .
$$

Setting $w=0$ in $(3.5),\left\langle S u_{n}, u_{n}\right\rangle+\left\langle T_{1} u_{n}, u_{n}\right\rangle \leqq\left\langle f, u_{n}\right\rangle \leqq$ const. for all $n$. Since $S$ is bounded, also $\left\langle T_{1} u_{n}, u_{n}\right\rangle \leqq$ const. for all $n$, and, by $\left(\mathrm{A}_{2}\right)$ and $\left(\mathrm{A}_{3}\right)$,

$$
u \in V_{1}, \quad \lambda_{1}(u) \leqq \liminf _{n}\left\langle T_{1} u_{n}, u_{n}\right\rangle \quad \text { and } \quad\left\langle T_{1} u_{n}, u_{m}\right\rangle \rightarrow\left\langle T_{1} u, u_{m}\right\rangle
$$

for any fixed $m$. Moreover, since $V^{*}$ is reflexive, there is $g \in V^{*}$ such that, by passing to a subsequence preserving the notation, $S u_{n} \rightarrow g$ weakly in $V^{*}$. For any fixed $m$ we then have

$$
\begin{aligned}
& \limsup _{n}\left\langle S u_{n}, u_{n}-u\right\rangle \leqq \limsup _{n}\left\langle S u_{n}, u_{n}-u_{m}\right\rangle+\limsup _{n}\left\langle S u_{n}, u_{m}-u\right\rangle \\
= & \limsup _{n}\left\{\left\langle T u_{n}, u_{n}-u_{m}\right\rangle-\left\langle T_{1} u_{n}, u_{n}-u_{m}\right\rangle\right\}+\left\langle g, u_{m}-u\right\rangle \\
\leqq & \limsup _{n}\left\langle T u_{n}, u_{n}-u_{m}\right\rangle-\liminf _{n}\left\{\left\langle T_{1} u_{n}, u_{n}\right\rangle-\left\langle T_{1} u_{n}, u_{m}\right\rangle\right\}+\left\langle g, u_{m}-u\right\rangle \\
\leqq & \limsup _{n}\left\langle f, u_{n}-u_{m}\right\rangle-\lambda_{1}(u)+\left\langle T_{1} u, u_{m}\right\rangle+\left\langle g, u_{m}-u\right\rangle .
\end{aligned}
$$


Letting $m \rightarrow \infty$ we obtain further by $\left(\mathrm{A}_{4}\right)$,

$$
\limsup _{n}\left\langle S u_{n}, u_{n}-u\right\rangle \leqq-\lambda_{1}(u)+\lambda_{1}(u)=0 .
$$

Since $S$ is pseudomonotone,

$$
\liminf _{n}\left\langle S u_{n}, u_{n}-w\right\rangle \geqq\langle S u, u-w\rangle \text { for all } w \in V .
$$

Therefore, by passing to a limit $n \rightarrow \infty$ in (3.5) with $w=w_{0}$,

$$
\left\langle T_{1} u, w_{0}\right\rangle-\lambda_{1}(u)+\left\langle S u, w_{0}-u\right\rangle \geqq\left\langle f, w_{0}-u\right\rangle .
$$

Since $w_{0}$ was arbitrary in $K \cap W$, (3.3) has been verified.

\section{Applications I}

Let $\Omega$ be an unbounded domain in $\mathbf{R}^{N}(N \geqq 2)$ and let $V=H_{0}^{k, p}(\Omega)$, $W=H_{0}^{k, s}(\Omega) \cap V$ with $0 \leqq m<k<N, 2 \leqq p<\infty$ and $s>\max \{p, N /(k-m)\}$. The space $W$ is furnished with the norm $\|\cdot\|_{W}=\max \left\{\|\cdot\|_{k, p},\|\cdot\|_{k, s}\right\}$; it then is a reflexive separable Banach space (see [9]). Since $s>N /(k-m), H_{0}^{k-\tau, s}(\Omega)$ is continuously embedded in $C(\Omega) \cap L^{\infty}(\Omega)$ for all $\tau \leqq m$ (see [8]); hence there is a constant $c>0$ such that for all $u \in H_{0}^{k-\tau, s}(\Omega)$,

$$
\|u\|_{\infty}=\sup _{x \in \Omega}|u(x)| \leqq c\|u\|_{k-\tau, s} .
$$

We impose on the operator $B u(x)=\sum_{|\beta| \leqq m}(-1)^{|\beta|} D^{\beta} B_{\beta}\left(x, \xi_{m}(u)(x)\right)$ the following conditions (cf. [2], [4], [11]).

$\left(\mathrm{B}_{1}\right) \quad B_{\beta}: \Omega \times \mathbf{R}^{s_{m}} \rightarrow \mathbf{R}$ satisfies Carathéodory's condition for all $|\beta| \leqq m$, i.e., $B_{\beta}\left(x, \xi_{m}\right)$ is measurable in $x$ for all $\xi_{m} \in \mathbf{R}^{s_{m}}$ and continuous in $\xi_{m}$ for almost all $x \in \Omega$;

$\left|B_{\beta}\left(x, \xi_{m}\right)\right| \leqq g(x) f\left(\xi_{m}\right)$ for almost all $x \in \Omega$ and all $\xi_{m} \in \mathbf{R}^{s_{m}}$ with $g \in L^{1}(\Omega)$, $f: \mathbf{R}^{s_{m}} \rightarrow R$ continuous;

Set

(B $\left.\mathbf{B}_{2}\right) \quad \Psi\left(x, \xi_{m}\right) \equiv \sum_{|\beta| \leqq m} B_{\beta}\left(x, \xi_{m}\right) \xi_{\beta} \geqq 0$ for almost all $x \in \Omega$ and all $\xi_{m} \in \mathbf{R}^{s_{m}}$.

$$
V_{1}=\left\{v \in V: B_{\beta}\left(\cdot, \xi_{m}(v)\right) \in L^{1}(\Omega) \text { for all }|\beta| \leqq m, \Psi\left(\cdot, \xi_{m}(v)\right) \in L^{1}(\Omega)\right\} .
$$

The semilinear form

$$
b(u, v)=\sum_{|\beta| \leqq m} \int_{\Omega} B_{\beta}\left(x, \xi_{m}(u)(x)\right) D^{\beta} v(x) d x
$$

is well-defined for all $u \in V_{1}$ and $v \in W$. Clearly

$$
|b(u, v)| \leqq \sum_{|\beta| \leqq m}\left\|B_{\beta}\left(\cdot, \xi_{m}(u)\right)\right\|_{1}\left\|D^{\beta} v\right\|_{\infty} \leqq c\|v\|_{W} \sum_{|\beta| \leqq m}\left\|B_{\beta}\left(\cdot, \xi_{m}(u)\right)\right\|_{1} .
$$


Hence for each $u \in V_{1}$ the map $v \rightarrow b(u, v)$ defines a continuous linear functional, $T_{1} u$ say, on $W$ by

$$
\left\langle T_{1} u, v\right\rangle=b(u, v), \quad v \in W .
$$

Obviously $T_{1}: D\left(T_{1}\right)=V_{1} \rightarrow W^{*}$ and $W \subset V_{1} \subset V$.

A further condition for $B$ is needed (cf. [2], [4], [11]).

$$
\left|B_{\beta}\left(x, \xi_{m}\right)\right| \leqq h\left(\left|\xi_{m}\right|\right) \Psi\left(x, \xi_{m}\right)+\sum_{|\alpha| \leqq m} \varphi_{\alpha}\left(x,\left|\xi_{\alpha}\right|\right),
$$

where we assume that $h: \mathbb{R}^{+} \rightarrow \mathbb{R}^{+}$is continuous, $h(r) \rightarrow 0$ as $r \rightarrow \infty$, and

$$
\varphi_{\alpha}(x, r)=\left\{\begin{array}{c}
K_{\alpha}(x) r^{s_{\alpha}} \text { with } K_{\alpha} \text { non-negative in } L^{\infty}(\Omega), \quad K_{\alpha}(x) \rightarrow 0 \text { as }|x| \rightarrow \infty, \\
S_{\alpha}(x) \Psi_{\alpha}(r) \text { with } K_{\alpha} \text { as above, } \Psi_{\alpha} \text { an Orlicz function satisfying the } \\
\quad \Delta^{2} \text {-condition, } \Psi_{\alpha}(r) \prec \Phi(r)=\left.|r|^{p} e^{|r|}\right|^{p^{\prime}} \text { at } 0 \text { and } \infty, \text { if } k-|\alpha|=N / p ; \\
K_{\alpha}(x) \theta_{\alpha}(r) \text { with } K_{\alpha} \text { non-negative in } L^{1}(\Omega) \text { and } \theta_{\alpha} ; \mathbb{R}^{+} \rightarrow \mathbb{R}^{+} \text {con- } \\
\text { tinuous, if } k-|\alpha|>N / p .
\end{array}\right.
$$

We remark that $\left(\mathrm{B}_{3}\right)$ automatically holds for $m=0$.

Lemma 4.1. If the conditions $\left(\mathrm{B}_{1}\right)-\left(\mathrm{B}_{3}\right)$ are satisfied, then the map $T_{1}: V_{1} \rightarrow W^{*}$ defined by (4.1) satisfies the conditions $\left(\mathrm{A}_{1}\right)-\left(\mathrm{A}_{3}\right)$ with $\lambda_{1}(u)=b(u, u)$.

Proof. We show first that $\left(\mathrm{A}_{2}\right)$ and $\left(\mathrm{A}_{3}\right)$ hold. To this end, let $\left(w_{n}\right) \subset W$ with $w_{n} \rightarrow v$ weakly in $V$, and let $\left\langle T_{1} w_{n}, w_{n}\right\rangle \leqq$ const. for all $n$. By Lemma 3 of [10] there is a subsequence $\left(v_{n}\right)$ of $\left(w_{n}\right)$ such that $D^{\alpha} v_{n}(x) \rightarrow D^{\alpha} v(x)$ a.e. in $\Omega$ for all $|\alpha| \leqq m$. By $\left(B_{1}\right), B_{\beta}\left(x, \xi_{m}\left(v_{n}\right)(x)\right) \rightarrow B_{\beta}\left(x, \xi_{m}(v)(x)\right)$ and $\Psi\left(x, \xi_{m}\left(v_{n}\right)(x)\right) \rightarrow \Psi\left(x, \xi_{m}(v)(x)\right)$ a.e. in $\Omega$. From $\left(B_{2}\right)$ it follows by Fatou's lemma that

$$
b(v, v)=\int_{\Omega} \Psi\left(x, \xi_{m}(v)(x)\right) d x \leqq \lim _{n} \inf \left\langle T_{1} v_{n}, v_{n}\right\rangle<\infty .
$$

By virtue of $\left(\mathrm{B}_{1}\right)$ and $\left(\mathrm{B}_{3}\right)$ there are positive constants $K_{1}$ and $K_{2}$ such that

$$
\left|B_{\beta}\left(x, \xi_{m}(v)(x)\right)\right| \leqq K_{1} \Psi\left(x, \xi_{m}(v)(x)\right)+\sum_{|\alpha| \leqq m} \varphi_{\alpha}\left(x,\left|D^{\alpha} v(x)\right|\right)+K_{2} g(x)
$$

for almost all $x \in \Omega$ and all $|\beta| \leqq m$. The embedding results of [8] imply (cf. [2]), taking account also of $\left(\mathrm{B}_{3}\right)$, that $B_{\beta}\left(., \xi_{m}(v)\right) \in L^{1}(\Omega)$ for all $|\beta| \leqq m$. Therefore $v \in V_{1}$.

To show that $T_{1} v_{n} \rightarrow T_{1} v$ weak ${ }^{*}$ in $W^{*}$ we invoke Vitali's convergence theorem (see, for example, [5], p. 150). Indeed, for any $z \in W$ we have

$$
\begin{gathered}
\left|\left\langle T_{1} v_{n}-T_{1} v, z\right\rangle\right| \leqq \sum_{|\beta| \leqq m} \int_{\Omega} \mid B_{\beta}\left(\left(x, \xi_{m}\left(v_{n}\right)(x)\right)-B_{\beta}\left(x, \xi_{m}(v)(x)\right)|| D^{\beta} z(x) \mid d x\right. \\
\leqq c\|z\|_{W} \sum_{|\beta| \leqq m} \int_{\Omega}\left|B_{\beta}\left(x, \xi_{m}\left(v_{n}\right)(x)\right)-B_{\beta}\left(x, \xi_{m}(v)(x)\right)\right| d x .
\end{gathered}
$$

Let $\delta>0, n$ and $x \in \Omega$ be given. Since $h(r) \rightarrow 0$ as $r \rightarrow \infty, h$ being continuous, there 
is a constant $K(\delta)$ such that $h(r) \leqq \delta$ if $r \geqq K(\delta)$. Therefore, either $\left|\xi_{m}\left(v_{n}\right)(x)\right| \leqq$ $K(\delta)$ or

$$
\left|B_{\beta}\left(x, \xi_{m}\left(v_{n}\right)(x)\right)\right| \leqq \delta \Psi\left(x, \xi_{m}\left(v_{n}\right)(x)\right)+\sum_{|\alpha| \leqq m} \varphi_{\alpha}\left(x,\left|D^{\alpha} v_{n}(x)\right|\right) .
$$

Let $K_{0}(\delta)=\sup \left\{\left|f\left(\xi_{m}\right)\right|:\left|\xi_{m}\right| \leqq K(\delta)\right\}<\infty$. For any measurable subset $E$ of $\Omega$,

$$
\begin{gathered}
\int_{E}\left|B_{\beta}\left(x, \xi_{m}\left(v_{n}\right)(x)\right)\right| d x \leqq K_{0}(\delta) \int_{E} g(x) d x+\delta \int_{E} \Psi\left(x, \xi_{m}\left(v_{n}\right)(x)\right) d x \\
+\sum_{|\alpha| \leqq m} \int_{E} \varphi_{\alpha}\left(x,\left|D^{\alpha} v_{n}(x)\right|\right) d x .
\end{gathered}
$$

By a further appeal to the embedding theorems of [8] we can see, as in [9], that the conditions of Vitali's theorem are met. In particular, for handling the terms $\varphi_{\alpha}$ involving exponentials we refer to the proof of Lemma 5.1 later on. Therefore we can conclude that $\left\langle T_{1} v_{n}, z\right\rangle \rightarrow\left\langle T_{1} v, z\right\rangle$ as $n \rightarrow \infty$. The same result for the initial sequence $\left(w_{n}\right)$ follows by a standard contradiction argument. Bearing in mind (4.2) we have then shown that $\left(\mathrm{A}_{2}\right)$ and $\left(\mathrm{A}_{3}\right)$ hold with $\lambda_{1}(v)=b(v, v)$.

To show finally that also $\left(A_{1}\right)$ holds it is clearly enough to prove that the restriction of $T_{1}$ to $W$ is demicontinuous. To this end let $w_{n} \rightarrow w$ in $W$. Then $w_{n} \rightarrow w$ also in $V$ and $\left\|D^{\alpha} w_{n}\right\|_{\infty} \leqq$ const. for all $n$. Hence by $\left(\mathrm{B}_{1}\right)$,

$$
\left|\left\langle T_{1} w_{n}, w_{n}\right\rangle\right| \leqq \sum_{|\beta| \leqq m} \int_{\Omega}\left|B_{\beta}\left(x, \xi_{m}\left(w_{n}\right)(x)\right)\right|\left|D^{\beta} w_{n}(x)\right| d x \leqq \text { const }\|g\|_{1} .
$$

That $T_{1} w_{n} \rightarrow T_{1} w$ weak ${ }^{*}$ in $W$, follows from the former part of the proof.

Example 4.2. Let

$$
A u(x)=\sum_{|\alpha|,|\beta| \leqq k}(-1)^{|\alpha|} D^{\alpha}\left(a_{\alpha \beta}(x) D^{\beta} u(x)\right),
$$

where the real measurable functions $a_{\alpha \beta}$ satisfy the following conditions (see [7]).

$$
\left\{\begin{aligned}
a_{\alpha \beta} \in L^{\infty}(\Omega) \text { if }|\alpha|=|\beta|=k & \\
M_{\mu(\alpha, \beta)}\left(\left|a_{\alpha \beta}\right|^{2}\right)<\infty & \text { for some } \mu(\alpha, \beta) \text { with } 0<\mu(\alpha, \beta)<2(k-\min \{|\alpha|,|\beta|\}), \\
& \text { if } \max \{|\alpha|,|\beta|\}=k,|\alpha|+|\beta|<2 k \\
M_{\mu(\alpha, \beta)}\left(\left|a_{\alpha \beta}\right|\right)<\infty & \text { for some } \mu(\alpha, \beta) \text { with } 0<\mu(\alpha, \beta)<2(k-\max \{|\alpha|,|\beta|\}), \\
& \text { if }|\alpha| \leqq k-1,|\beta| \leqq k-1 .
\end{aligned}\right.
$$

For $V=H_{0}^{k, 2}(\Omega)$ it is a routine application of the embedding theorems of [7] to verify that $A$ gives rise to a bounded linear map $S: V \rightarrow V^{*}$ by the rule

$$
\langle S u, v\rangle=a(u, v) \equiv \sum_{|\alpha|,|\beta| \leqq k} \int_{\Omega} a_{\alpha \beta}(x) D^{\beta} u(x) D^{\alpha} v(x) d x .
$$

Moreover, if

$$
\sum_{|\alpha|,|\beta| \leqq k} a_{\alpha \beta} \xi_{\alpha} \xi_{\beta} \geqq 0 \text { for all } x \in \Omega \text { and } \xi_{k} \in \mathbf{R}^{s_{k}},
$$

holds, then $S$ is monotone. In view of Corollary 3.2 we obtain the following 
Theorem 4.3. Let the conditions $\left(\mathrm{B}_{1}\right)-\left(\mathrm{B}_{3}\right),\left(\mathrm{C}_{1}\right)$ and $\left(\mathrm{C}_{2}\right)$ be satisfied, and let $K$ be a closed convex subset of $V$ containing the origin. If for a given $f \in V^{*}$ and for some $R>0$,

$$
a(w, w)+b(w, w)-\langle f, w\rangle>0 \quad \text { for all } w \in K \cap W, \quad\|w\|_{V}=R,
$$

then the variational inequality

$$
a(u, w-u)+b(u, w-u) \geqq\langle f, w-u\rangle \text { for all } w \in K \cap W,
$$

admits a solution $u$ in $K \cap V_{1}$.

Remark 4.4. The inequality (4.5) is solvable in particular, if the bilinear form $a(.,$.$) is coercive, i.e. there is c_{0}>0$ such that

$$
a(u, u) \geqq c_{0}\|u\|_{V}^{2} \text { for all } u \in V .
$$

Since $b(u, u) \geqq 0$ for all $u \in W$ by $\left(\mathrm{B}_{2}\right),(4.4)$ clearly holds.

To apply Theorem 3.3 for situations where $A$ is a quasilinear operator, we impose on $B$ the following further condition (cf. [2], [4], [11]).

$\left(\mathrm{B}_{4}\right)$ For each $\varepsilon>0$ there is a constant $K_{\varepsilon}>0$ such that for almost all $x \in \Omega$, all $\xi_{m}, \xi_{m}^{*} \in \mathbf{R}^{s_{m}}$ and $|\beta| \leqq m$,

$$
B_{\beta}\left(x, \xi_{m}\right) \xi_{\beta}^{*} \leqq \varepsilon \Psi\left(x, \xi_{m}^{*}\right)+K_{\varepsilon}\left\{\bar{g}(x)+\Psi\left(x, \xi_{m}\right)+\sum_{|\alpha| \leqq m}\left[\varphi_{\alpha}\left(x,\left|\xi_{\alpha}\right|\right)+\varphi_{\alpha}\left(x,\left|\xi_{\alpha}^{*}\right|\right)\right]\right\}
$$

with $\bar{g} \in L^{1}(\Omega)$.

Lemma 4.5 If the conditions $\left(\mathrm{B}_{1}\right)-\left(\mathrm{B}_{4}\right)$ are satisfied, then the map $T_{1}: V_{1} \rightarrow W^{*}$ defined by (4.1) satisfies the conditions $\left(\mathrm{A}_{1}\right)-\left(\mathrm{A}_{4}\right)$ with $\lambda_{1}(u)=b(u, u)$.

Proof. In view of Lemma 4.1 we must only show that $\left(\mathrm{A}_{4}\right)$ holds. Let $\left(v_{n}\right)$ be the sequence occurring in the proof of Lemma 4.1, and let $[t]^{+}$stand for max $\{0, t\}$, $t \in \mathbf{R}$. It follows from $\left(\mathrm{B}_{2}\right)$ that

$$
\left[\sum_{|\beta| \leqq m} B_{\beta}\left(x, \xi_{m}(v)(x)\right) D^{\beta} v_{n}(x)\right]^{+} \rightarrow \Psi\left(x, \xi_{m}(v)(x)\right) \quad \text { a.e. on } \quad \Omega .
$$

By $\left(\mathrm{B}_{4}\right)$, for each $\varepsilon>0$ there is $K_{\varepsilon}>0$ such that

$$
\begin{gathered}
\frac{1}{s_{m}}\left[\sum_{\beta \mid \leqq m} B_{\beta}\left(x, \xi_{m}(v)(x)\right) D^{\beta} v_{n}(x)\right]^{+} \leqq \varepsilon \Psi\left(x, \xi_{m}\left(v_{n}\right)(x)\right) \\
+K_{\varepsilon}\left\{\bar{g}(x)+\Psi\left(x, \xi_{m}(v)(x)\right)+\sum_{|\alpha| \leqq m}\left[\varphi_{\alpha}\left(x,\left|D^{\alpha} v(x)\right|\right)+\varphi_{\alpha}\left(x,\left|D^{\alpha} v_{n}(x)\right|\right)\right]\right\},
\end{gathered}
$$

where $\bar{g}, \Psi\left(., \xi_{m}(v)\right)$ and $\varphi_{\alpha}\left(.,\left|D^{\alpha} v\right|\right)$ are in $L^{1}(\Omega)$. By an appeal to Vitali's convergence theorem as in the proof of Lemma 4.1 we can conclude that

$$
\int_{\Omega}\left[\sum_{|\beta| \leqq m} B_{\beta}\left(x, \xi_{m}(v)(x)\right) D^{\beta} v_{n}(x)\right]^{+} d x \rightarrow b(v, v)=\lambda_{1}(v) \quad \text { as } \quad n \rightarrow \infty .
$$

Therefore, $\lim \sup \left\langle T_{1} v, v_{n}\right\rangle \leqq \lambda_{1}(v)$. 
Example 4.6. Let $A u(x)=\sum_{|\alpha| \leqq k}(-1)^{|\alpha|} D^{\alpha} A_{\alpha}\left(x, \xi_{k}(u)(x)\right), 0 \leqq m<k<N$ and $V=H_{0}^{k, p}(\Omega)$ with $2 \leqq p<\infty$. Under certain conditions for the functions $A_{\alpha}: \Omega \times$ $\times \mathbf{R}^{s_{k}} \rightarrow \mathbf{R}$, which can be found in [10], $A$ gives rise to a pseudomonotone map $S: V \rightarrow V^{*}$ by the rule

$$
\langle S u, v\rangle=c(u, v) \equiv \sum_{|\alpha| \leqq k} \int_{\Omega} A_{\alpha}\left(x, \xi_{k}(u)(x)\right) D^{\alpha} v(x) d x, \quad u, v \in V .
$$

According to Theorem 3.3 we then have

Theorem 4.7. Let the conditions $\left(\mathrm{B}_{1}\right)-\left(\mathrm{B}_{4}\right)$ be satisfied, let $S: V \rightarrow V^{*}$ be a pseudomonotone map defined by (4.6), and let $K$ be a closed convex subset of $V$ containing the origin. If for a given $f \in V^{*}$ and for some $R>0$,

$$
c(w, w)+b(w, w)-\langle f, w\rangle>0 \text { for all } w \in K \cap W, \quad\|w\|_{V}=R,
$$

then the variational inequality

$$
c(u, w-u)+b(u, w-u) \geqq\langle f, w-u\rangle \text { for all } w \in K \cap W,
$$

admits a solution $u$ in $K \cap V_{1}$.

\section{Orlicz space method}

In the sequel we focus our attention on the particular case $V=H_{0}^{k, 2}(\Omega), 0 \leqq m<$ $<k<N$ and $k-m=N / 2$, where $A$ is a linear differential operator as in Example 4.2. We shall show that the sign condition $\left(\mathrm{B}_{2}\right)$ can be weakened or removed by replacing the condition $\left(\mathrm{B}_{4}\right)$ with a rather mild growth condition.

Let $\Omega$ be an unbounded domain in $\mathbb{R}^{N}(N \geqq 2)$. To introduce the strongly nonlinear operator $B$ we set the following conditions for the functions $B_{\beta}: \Omega \times \mathbf{R}^{s_{m}} \rightarrow \mathbf{R}$, $|\beta| \leqq m$.

$\left(D_{1}\right) \quad B_{\beta}$ satisfies Carathéodory's condition for all $|\beta| \leqq m$ and $B_{\beta}(x, 0)=0$ for almost all $x \in \Omega$ and all $|\beta| \leqq m$;

$\left(\mathrm{D}_{2}\right) \quad\left|B_{\beta}\left(x, \xi_{m}\right)\right| \leqq C\left(\xi_{m-1}\right)\left\{P(x)+Q(x) \sum_{|\alpha|=m} \Psi_{\alpha}\left(\xi_{\alpha}\right)\right\}$ for almost all $x \in \Omega$ and all $\xi_{m} \in \mathbf{R}^{s_{m}}$, where $C: \mathbf{R}^{s_{m-1} \rightarrow \mathbf{R}}$ is continuous, $P$ and $Q$ are non-negative functions with $P \in L^{2}(\Omega), \int_{\Omega}\left|P_{R}(x)\right|^{2} d x \rightarrow 0$ as $R \rightarrow \infty, Q \in L^{\infty}(\Omega), Q(x) \rightarrow 0$ as $|x| \rightarrow \infty$ in $\Omega$, and $\Psi_{\alpha},|\alpha|=m$, are Orlicz functions satisfying the $\Delta^{2}$-condition and $\Psi_{\alpha} \prec \Phi_{0}$ at 0 and $\infty$ with $\Phi_{0}(r)=r^{2} e^{r^{2}}$.

To show that the semilinear form

$$
b(u, v)=\sum_{|\beta| \leqq m} \int_{\Omega} B_{\beta}\left(x, \xi_{m}(u)(x)\right) D^{\beta} v(x) d x
$$

is bounded on $V \times V$, we recall that $H_{0}^{k-|\alpha|, 2}(\Omega)$ is continuously embedded in 
$C(\Omega) \cap L^{\infty}(\Omega)$ for all $|\alpha| \leqq m-1$, and $H_{0}^{k-m, 2}(\Omega)$ in $L_{\Phi_{0}}(\Omega)$ (see [8]). By $\left(\mathrm{D}_{2}\right)$ we have

$$
\begin{gathered}
|b(u, v)| \\
\leqq \sum_{|\beta| \leqq m} \int_{\Omega}\left|C\left(\xi_{m-1}(u)(x)\right)\right|\left\{P(x)\left|D^{\beta} v(x)\right|+Q(x) \sum_{|\alpha|=m} \Psi_{\alpha}\left(D^{\alpha} u(x)\right)\left|D^{\beta} v(x)\right|\right\} d x .
\end{gathered}
$$

Since $C$ is continuous we get, by taking account of the embeddings above, that

$$
\left|C\left(\xi_{m-1}(u)(x)\right)\right| \leqq h\left(\|u\|_{k, 2}\right) \text {. for all } x \in \Omega,
$$

where $h: \mathbb{R}^{+} \rightarrow \mathbf{R}^{+}$is a continuous non-decreasing function. Therefore, by the Cauchy-Schwarz inequality,

$$
|b(u, v)| \leqq h\left(\|u\|_{k, 2}\right)\left\{\|P\|_{2}+\|Q\|_{\infty} \sum_{|\alpha|=m}\left\|\Psi_{\alpha}\left(D^{\alpha} u\right)\right\|_{2}\right\} \sum_{|\beta| \leqq m}\left\|D^{\beta} v\right\|_{2} .
$$

Since $\Psi_{\alpha} \prec \Phi_{0}$ at 0 and $\infty$ and since $\Psi_{\alpha}$ satisfies the $\Delta^{2}$-condition, also $\Psi_{\alpha}^{2} \prec \Phi_{0}$ at 0 and $\infty$ for all $|\alpha|=m$. Hence for each $\varepsilon>0$ a constant $K(\varepsilon)$ can be found such that

$$
\int_{\Omega} \Psi_{\alpha}^{2}\left(D^{\alpha} u(x)\right) d x \leqq K(\varepsilon) \int_{\Omega} \Phi_{0}\left(\varepsilon D^{\alpha} u(x)\right) d x .
$$

Choosing $\varepsilon$ so small that $\left\|\varepsilon D^{\alpha} u\right\|_{\Phi_{0}} \leqq 1$ we obtain that

$$
\int_{\Omega} \Psi_{\alpha}^{2}\left(D^{\alpha} u(x)\right) d x \leqq K(\varepsilon) \varepsilon\left\|D^{\alpha} u\right\|_{\Phi_{0}} \leqq \text { const }\|u\|_{k, 2} \cdot
$$

Consequently, there is a constant $K_{0}>0$ such that for all $u, v \in V$,

$$
|b(u, v)| \leqq K_{0} h\left(\|u\|_{k, 2}\right)\left\{1+\|Q\|_{\infty}\|u\|_{k, 2}^{1 / 2}\right\}\|v\|_{k, 2} .
$$

Thus it is clear that $b$ gives rise to a bounded map $T_{1}: V \rightarrow V^{*}=V$ by the rule

$$
\left\langle T_{1} u, v\right\rangle=b(u, v), \quad u, v \in V
$$

Lemma 5.1. If the conditions $\left(\mathrm{D}_{1}\right)$ and $\left(\mathrm{D}_{2}\right)$ are satisfied, then the map $T_{1}: V \rightarrow V$ defined by (5.3) is compact and continuous.

Proof. Let $\left(u_{i}\right)$ be a bounded sequence in $V, R>0, \Omega_{R}=\Omega \cap B(0, R)$, and let $\chi_{R}$ stand for the characteristic function of $B(0, R)$. Then

$$
\begin{aligned}
\left\|T_{1} u_{i}-T_{1} u_{j}\right\| & =\sup _{\|v\|_{k, 2}=1}\left|\sum_{|\beta| \leqq m} \int_{\Omega}\left\{B_{\beta}\left(x, \xi_{m}\left(u_{i}\right)(x)\right)-B_{\beta}\left(x, \xi_{m}\left(u_{j}\right)(x)\right)\right\} D^{\beta} v(x) d x\right| \\
& \leqq \sum_{|\beta| \leqq m}\left\|B\left(\cdot, \xi_{m}\left(u_{i}\right)\right)-B_{\beta}\left(\cdot, \xi_{m}\left(u_{j}\right)\right)\right\|_{2} .
\end{aligned}
$$


By $\left(\mathrm{D}_{1}\right), \chi_{R}(x) B_{\beta}\left(x, \xi_{m}(u)(x)\right)=B_{\beta}\left(x, \xi_{m}\left(\chi_{R} u\right)(x)\right)$ so that

$$
\begin{gathered}
\int_{\Omega}\left|B_{\beta}\left(x, \xi_{m}\left(u_{i}\right)(x)\right)-B_{\beta}\left(x, \xi_{m}\left(u_{j}\right)(x)\right)\right|^{2} d x \\
\leqq 9\left\{\int_{\Omega_{R}}\left|B_{\beta}\left(x, \xi_{m}\left(\chi_{R} u_{i}\right)(x)\right)-B_{\beta}\left(x, \xi_{m}\left(\chi_{R} u_{j}\right)(x)\right)\right|^{2} d x\right. \\
\left.+\int_{\Omega}\left|\left(1-\chi_{R}(x)\right) B_{\beta}\left(x, \xi_{m}\left(u_{i}\right)(x)\right)\right|^{2} d x+\int_{\Omega}\left|\left(1-\chi_{R}(x)\right) B_{\beta}\left(x, \xi_{m}\left(u_{j}\right)(x)\right)\right|^{2} d x\right\} \\
=J_{R}(i, j)+J_{R}(i)+J_{R}(j) .
\end{gathered}
$$

Here $u \rightarrow \chi_{R} u$ is a compact map from $H_{0}^{k-\alpha, 2}(\Omega)$ to $L^{2}(\Omega)$ for all $|\alpha| \leqq m-1$ and from $H_{0}^{k-m, 2}(\Omega)$ to any $E_{\Psi}(\Omega)$ with $\Psi \prec \Phi_{0}$ at 0 and $\infty$ (see [8]). Hence there is a subsequence of $\left(u_{i}\right)$, still denoted by $\left(u_{i}\right)$, such that $\left(\chi_{R}(x) D^{x} u_{i}(x)\right)$ converges in measure on $\Omega_{R}$ for all $|\alpha| \leqq m$. By $\left(D_{1}\right)$ it follows from Nemytskii's lemma (see [13], p. 20) that also $\left\{B_{\beta}\left(x, \xi_{m}\left(\chi_{R} u_{i}\right)(x)\right)\right\}$ converges in measure on $\Omega_{R}$. In view of the now familiar Vitali convergence theorem, $J_{R}(i, j) \rightarrow 0$ as $i, j \rightarrow \infty$, if we can show that the family $\left\{\left|B_{\beta}\left(., \xi_{m}\left(u_{i}\right)\right)\right|^{2}\right\}$ has absolutely equicontinuous integrals on $\Omega_{R}$. Indeed, by $\left(\mathrm{D}_{2}\right)$,

$$
\mid B_{\beta}\left(x,\left.\xi_{m}\left(u_{i}(x)\right)\right|^{2} \leqq \text { const }\left\{|P(x)|^{2}+\|Q\|_{\infty}^{2} \sum_{\alpha=m}\left|\Psi_{\alpha}\left(D^{\alpha} u_{i}(x)\right)\right|^{2}\right\}\right.
$$

for all $|\beta| \leqq m$ and almost all $x \in \Omega_{R}$. Since $P \in L^{2}(\Omega)$ we need only to deal with the family $\left\{\left|\Psi_{\alpha}\left(D^{\alpha} u_{i}(x)\right)\right|^{2}\right\}$. By the $\Delta^{2}$-condition, $\Psi_{\alpha}^{4}<\Phi_{0}$ at 0 and $\infty$, for example. Now we can invoke Vallée-Poussin's theorem (see [14], p. 94). Indeed, for an $\varepsilon>0$ small enough,

$$
\begin{aligned}
\int_{\Omega_{R}}\left|\Psi_{\alpha}\left(D^{\alpha} u_{i}(x)\right)\right|^{4} d x & \leqq K(\varepsilon) \int_{\Omega_{R}} \Phi_{0}\left(\varepsilon D^{\alpha} u_{i}(x)\right) d x \leqq K(\varepsilon)\left\|\varepsilon D^{\alpha} u_{i}\right\|_{\Phi_{0}} \\
& \leqq \text { const }\left\|u_{i}\right\|_{k, 2} \leqq L
\end{aligned}
$$

for some positive constant $L$. By using the same argument for all $|\alpha|=m$ we can conclude that $J_{R}(i, j) \rightarrow 0$ as $i, j \rightarrow \infty$. Similarly, since $Q(x) \rightarrow 0$ as $|x| \rightarrow \infty$ in $\Omega$,

$$
\begin{aligned}
J_{R}(i) & \leqq \text { const } \int_{\Omega}\left\{\left|P_{R}(x)\right|^{2}+\left|Q_{R}(x)\right|^{2} \sum_{|\alpha|=m}\left|\Psi_{\alpha}\left(D^{\alpha} u_{i}(x)\right)\right|^{2}\right\} d x \\
& \leqq \text { const }\left\{\int_{\Omega}\left|P_{R}(x)\right|^{2} d x+\operatorname{const} \delta(R)\right\},
\end{aligned}
$$

where $\delta(R) \rightarrow 0$ as $R \rightarrow \infty$. By $\left(\mathrm{D}_{2}\right)$ we then have that $J_{R}(i) \rightarrow 0$ as $R \rightarrow \infty$, and by the same argument $J_{R}(j) \rightarrow 0$ as $R \rightarrow \infty$. Since all terms may be handled in the similar manner, it is now clear that for any $\varepsilon>0$ a subsequence of $\left(u_{i}\right)$ and a natural number $i_{0}$ can be found such that

$$
\left\|T_{1} u_{i}-T_{1} u_{j}\right\|<\varepsilon \quad \text { whenever } \quad i, j \geqq i_{0} .
$$

Thus the compactness of $T_{1}$ has been proved. The continuity follows in the same way. 
Remark 5.2. For $m=0$ and $N=2 k, B u(x)=B_{0}(x, u(x))$. In [8] Lemma 5.1 is proved assuming that $B_{0}(x, r)=Q(x) \psi(r)$, where $Q \in L^{\infty}(\Omega), Q(x) \rightarrow 0$ as $|x| \rightarrow \infty$ in $\Omega, \Psi \prec \Phi_{0}$ at 0 and $\infty, \Psi$ satisfies the $\Delta_{3}$-condition and $\widetilde{\Psi}$ (the complementary Orlicz function) satisfies the $\Delta_{2}$-condition for all $r \geqq 0$. We note that for $\Psi$ satisfying the $\Delta^{2}$-condition, the $\Delta_{3}$-condition holds for large values of $r$ only (see [14]).

We are now in a position to produce the solvability theorems for variational inequalities involving the maps $T=S+T_{1}: V \rightarrow V$, where $S$ is a bounded linear and monotone map induced by (4.3).

Theorem 5.3. Let the conditions $\left(\mathrm{C}_{1}\right),\left(\mathrm{C}_{2}\right),\left(\mathrm{D}_{1}\right)$ and $\left(\mathrm{D}_{2}\right)$ be satisfied and let $K$ be a closed convex subset of $V$ containing the origin. If for a given $f \in V$ and for some $R>0$,

$$
\langle S v, v\rangle+\left\langle T_{1} v, v\right\rangle-\langle f, v\rangle>\cup \quad \text { for all } \quad v \in K \cap V, \quad\|v\|_{k, 2}=R,
$$

then the variational inequality

$$
\langle S u, v-u\rangle+\left\langle T_{1} u, v-u\right\rangle \geqq\langle f, v-u\rangle \text { for all } v \in K \cap V,
$$

admits a solution $u$ in $K \cap V$.

Proof. The assertion follows immediately from Corollary 3.2, because $T_{1}$, being a compact and continuous map from $V$ to $V^{*}$, satisfies the conditions $\left(\mathrm{A}_{1}\right)-\left(\mathrm{A}_{3}\right)$ with $\lambda_{1}(u)=\left\langle T_{1} u, u\right\rangle$.

Next we consider the case where the condition $\left(C_{2}\right)$ is replaced by $\left(C_{3}\right)$, i.e. the bilinear form $a(.,$.$) is coercive. First we introduce a condition$

$\left(\mathrm{D}_{3}\right) \quad \Psi\left(x, \xi_{m}\right)=\sum_{|\beta| \leqq m} B_{\beta}\left(x, \xi_{m}\right) \xi_{\beta} \geqq-F(x)$ for almost all $x \in \Omega$ and all $\xi_{m} \in \mathbf{R}^{s_{m}}$ with $F \in L^{1}(\Omega)$.

Theorem 5.4. Let the conditions $\left(\mathrm{C}_{1}\right),\left(\mathrm{C}_{3}\right),\left(\mathrm{D}_{1}\right)-\left(\mathrm{D}_{3}\right)$ be satisfied and let $K$ be a closed convex subset of $V$ containing the origin. Then the variational inequality (5.5) admits a solution $u$ in $K \cap V$ for any $f \in V$.

Proof. By the conditions $\left(\mathrm{C}_{3}\right)$ and $\left(\mathrm{D}_{3}\right)$ we have that

$$
\|v\|_{k, 2}^{-1}\left\{\langle S v, v\rangle+\left\langle T_{1} v, v\right\rangle\right\} \geqq c_{0}\|v\|_{k, 2}-\|F\|_{1} \rightarrow \infty \quad \text { as } \quad\|v\|_{k, 2} \rightarrow \infty .
$$

Therefore (5.4) holds for some $R>0$ and for any $f \in V$. The assertion follows from Theorem 5.3.

The following theorem utilises the estimate (5.2). Since $h: \mathbf{R}^{+} \rightarrow \mathbf{R}^{+}$is continuous and non-decreasing, $\lim \sup _{r \rightarrow \infty} h(r) r^{-1 / 2}=\gamma$ with $0 \leqq \gamma \leqq \infty$.

Theorem 5.5. Let the conditions $\left(\mathrm{C}_{1}\right),\left(\mathrm{C}_{3}\right),\left(\mathrm{D}_{1}\right)$ and $\left(\mathrm{D}_{2}\right)$ be satisfied and let $K$ be a closed convex subset of $V$ containing the origin. If $\gamma=0$, the variational inequality (5.5) admits a solution $u$ in $K \cap V$ for any $f \in V$. If $0<\gamma<\infty$, then (5.5) admits a solution $u$ in $K \cap V$ for any $f \in V$ provided $\|Q\|_{\infty}$ is small enough. 
Proof. By (5.2) we have

Hence

$$
\langle S v, v\rangle+\left\langle T_{1} v, v\right\rangle \geqq c_{0}\|v\|_{k, 2}^{2}-K_{0} h\left(\|v\|_{k, 2}\right)\left\{1+\|Q\|_{\infty}\|v\|_{k, 2}^{1 / 2}\right\}\|v\|_{k, 2} .
$$

$$
\|v\|_{k, 2}^{-1}\left\{\langle S v, v\rangle+\left\langle T_{1} v, v\right\rangle\right\} \geqq\|v\|_{k, 2}\left\{c_{0}-K_{0}\|Q\|_{\infty} \frac{h\left(\|v\|_{k, 2}\right)}{\|v\|_{k, 2}^{1 / 2}}-K_{0} \frac{h\left(\|v\|_{k, 2}\right)}{\|v\|_{k, 2}}\right\} .
$$

If $\gamma=0,(5.4)$ obviously holds for some $R>0$ and for any $f \in V$. If $0<\gamma<\infty$, (5.4) holds provided that $\|Q\|_{\infty}<c_{0} K_{0}^{-1} \gamma^{-1}$.

For the particular case $m=0, N=2 k$, when $C \equiv 1$ in $\left(\mathrm{D}_{2}\right)$ and $h(r) \equiv 1$ in (5.2), we obtain the following result, which at the cost of the growth condition $\left(D_{2}\right)$ relaxes the sign condition for the function $B_{0}: \Omega \times \mathbf{R} \rightarrow \mathbf{R}$. We remind that the map $T_{1}$ is now defined by

$$
\left\langle T_{1} u, v\right\rangle=\int_{\Omega} B_{0}(x, u(x)) v(x) d x, \quad u, v \in V .
$$

Corollary 5.6. Let the conditions $\left(\mathrm{C}_{1}\right),\left(\mathrm{C}_{3}\right),\left(\mathrm{D}_{1}\right)$ and $\left(\mathrm{D}_{2}\right)$ be satisfied and let $K$ be a closed convex subset of $H_{0}^{k, 2}(\Omega)(k=N / 2)$ containing the origin. Then the variational inequality (5.5) admits a solution u in $K \cap H_{0}^{k, 2}(\Omega)$ for any $f \in H_{0}^{k, 2}(\Omega)$.

Remark 5.7. If $A$ satisfies an ellipticity condition

$$
\sum_{|\alpha|=|\beta|=k} a_{\alpha \beta}(x) \xi^{\alpha} \xi^{\beta} \geqq c_{1}|\xi|^{2 k} \text { for all } x \in \Omega \text { and } \xi \in \mathbf{R}^{N} \text {, }
$$

$c_{1}>0$ a constant, and if Poincare's inequality holds in $H_{0}^{k, 2}(\Omega)$; for instance, $\Omega \subset \mathbf{R}^{2 k}$ is an infinite strip, then $\left(\mathrm{C}_{3}\right)$ holds provided the lower order terms of $A$ are not too large in a certain technical sense (see [7], Lemma 5.2).

\section{Applications II}

We shall apply the solvability theorems of $\S 5$ for a strongly nonlinear elliptic Dirichlet problem

$$
\left\{\begin{array}{l}
A u(x)+B u(x)=F(x) \text { in } \Omega \\
D^{\alpha} u(x)=0 \text { on } \partial \Omega \text { for all }|\alpha| \leqq k-1,
\end{array}\right.
$$

where $\Omega \subset \mathbf{R}^{N}$ ( $\left.N \geqq 2\right)$ is an unbounded domain, $A$ satisfies the conditions $\left(\mathrm{C}_{1}\right)$ and $\left(\mathrm{C}_{2}\right)$ or $\left(\mathrm{C}_{3}\right), B$ satisfies $\left(\mathrm{D}_{1}\right)$ and $\left(\mathrm{D}_{2}\right), F \in L^{2}(\Omega)$ and $0 \leqq m<k<N$ with $k-m=$ $=N / 2$. For any $F \in L^{2}(\Omega)$ there is an element $f \in V=H_{0}^{k, 2}(\Omega)$ such that

$$
\int_{\Omega} F(x) v(x) d x=\langle f, v\rangle \text { for all } v \in V .
$$


A variational (weak) solution of (6.1) is any $u \in V$ such that

$$
a(u, v)+b(u, v)=\langle f, v\rangle \text { for all } v \in V,
$$

where $a$ and $b$ are defined by (4.3) and (5.1) respectively. Therefore, by a choice $K=V$, the solvability of the variational inequality (5.5) guarantees the existence of a solution of (6.2). Thus the theorems for there to exist a solution of (6.2) are particular cases of the solvability theorems of $\S 5$. One can see that these results generalise the corresponding results obtained in [6] and [8].

As a particular example of an equation which can be handled by this method we cite

$$
-\Delta u(x)+P(x) \sin u(x)+Q(x)|u(x)|^{\varrho} e^{|u(x)|^{\delta}}=F(x), \quad x \in \Omega,
$$

where $\Omega \subset \mathbb{R}^{2}$ such that Poincare's inequality holds in $H_{0}^{1,2}(\Omega), P \in L^{2}(\Omega), Q \in L^{\infty}(\Omega)$, $P(x), Q(x) \rightarrow 0$ as $|x| \rightarrow \infty$ in $\Omega \quad \varrho>2,1 \leqq \delta<2$. For strongly nonlinear eigenvalue problems of the type (6.1) we refer to [16].

Acknowledgements. The writer wishes to thank Professor D. E. Edmunds for numerous stimulating conversations and for his valuable advice. The financial support provided by the British Council is gratefully acknowledged.

\section{References}

[1] BRowder, F. E.: On the unification of the calculus of variations and the theory of monotone nonlinear operators in Banach spaces. - Proc. Nat. Acad. Sci. U.S.A. 56. 1966, 419425.

[2] BRowder, F. E.: Existence theory for boundary value problems for quasilinear ellipticsystems with strongly nonlinear lower order terms. - Proc. Sympos. Pure Math., Vol. 23, Amer. Math. Soc., 1973, 269-286.

[3] Browder, F. E., and P. Hess: Nonlinear mappings of monotone type in Banach spaces. - J. Functional Analysis. 11, 1972, 251-294.

[4] CÁc, N. P.: On strongly nonlinear variational inequalities. - J. Math. Pures Appl. 57, 1975 , $1-10$.

[5] Dunford, N., and J. T. Schwartz: Linear operators, Part I. - Interscience Publishers, Inc., New York, 1958.

[6] Edmunds, D. E.: Nonlinear elliptic equations. - Proc. Conf. on Ordinary and Partial Differential equations, Dundee, Lecture Notes in Mathematics, vol. 415, 1974.

[7] Edmunds, D. E., and W. D. Evans: Elliptic and degenerate-elliptic operators in unbounded domains. - Ann. Scuola Norm. Sup. Pisa Sci. Fis. Mat. 27, 1973, 591-640.

[8] Edmunds, D. E., and W. D. Evans: Orlicz spaces on unbounded domains. - Proc. Roy. Soc. London Ser. A 342, 1975, 373-400.

[9] Edmunds, D. E., V. B. Moscatelli, and J. R. L. Webb: Strongly nonlinear elliptic operators in unbounded domains. - Publ. Math. Bordeaux, Fasc. 4.

[10] Edmunds, D. E., and J. R. L. WebB: Quasilinear elliptic problems in unbounded domains. Proc. Roy. Soc. London Ser. A 334, 1973, 397-410.

[11] Hess, P.: Variational inequalities for strongly nonlinear elliptic operators. - J. Math. Pures. Appl. 52, 1973, 285-298.

[12] Hess, P.: On a class of strongly nonlinear elliptic variational inequalities. - Math. Ann. 211. $1974,289-297$. 
[13] Krasnosel'skir, M. A.: Topological methods in the theory of nonlinear integral equations. Pergamon Press, Oxford-London-New York-Paris, 1964.

[14] Krasnosel'skiI, M. A., and YA. B. Rutickir: Convex functions and Orlicz Spaces. - P. Noordhoff Ltd., Groningen, 1961.

[15] Lions, J. L.: Quelques méthodes de résolution des problèmes aux limites non linéaires. Dunod, Gauthier-Villars, Paris, 1969.

[16] Mustonen, V.: Strongly nonlinear eigenvalue problems. - Quart. J. Math. Oxford (2) 27. 1976, 489-509.

University of Oulu

Department of Mathematics

SF-90570 Oulu 57

Finland

Received 14 November 1975
University of Sussex

School of Mathematics

Brighton BN1 9QH

England 